\title{
FITOMASSA AÉREA RESIDUAL DA PASTAGEM DE INVERNO NO SISTEMA INTEGRAÇÃO LAVOURA-PECUÁRIA
}

\section{AERIAL BIOMASS RESIDUE OF WINTER ANNUAL FORAGES IN AN INTEGRATED CROP-LIVESTOCK SYSTEM}

\author{
Claudete Reisdorfer LANG ${ }^{1}$ \\ Adelino PELISSARI ${ }^{2}$ \\ Anibal DE MORAES ${ }^{2}$ \\ Reuben Mark SULC ${ }^{3}$ \\ Edilson Batista de OLIVEIRA ${ }^{4}$ \\ Paulo César de Faccio CARVALHO 5
}

\begin{abstract}
RESUMO
Produtores no Sul do Brasil estão procurando diversificar a propriedade aumentando a eficiência de produção através da integração lavoura-pecuária. O objetivo foi determinar o efeito do pastejo e da adubação nitrogenada na decomposição dos resíduos da pastagem anual de inverno no milho. A cobertura de Avena strigosa Schreb mais Lolium multiflorum L. foi semeada em abril de 2002, em Ponta Grossa - PR. A combinação de tratamentos com e sem nitrogênio, com e sem pastejo foi aplicada sobre a pastagem de inverno. O período de pastejo ocorreu de junho à outubro de 2002, a pastagem foi dessecada em 11 de outubro e o milho semeado vinte dias depois, tendo sido aplicados duas doses de nitrogênio $\left(0\right.$ e $\left.150 \mathrm{~kg} \cdot \mathrm{ha}^{-1}\right)$. A pastagem de inverno, cortada, foi colocada em sacos de nylon que foram espalhados sobre o solo, e recolhidos em cinco épocas com intervalos de três semanas para análise de matéria seca residual. Os dados foram submetidos a análise de variância. Ajustes de modelo matemático indicaram que a redução da massa seca ocorreu de acordo com o modelo quadrático em função do tempo. A decomposição dos resíduos da pastagem foi afetada pelos tratamentos impostos $(p<0,02)$, pelas doses de nitrogênio no milho $(p<0,01)$ e suas interações. Na última data de avaliação (132 dias do período experimental) foram verificadas diferenças $(p<0,01)$ apenas para os tratamentos sobre a pastagem de inverno. Tratamentos que receberam adubação nitrogenada no inverno resultaram em decomposição maior nas áreas com e sem pastejo.
\end{abstract}

Palavras-chave: pastejo, nitrogênio, decomposição, Zea mays L.

\begin{abstract}
Producers in southern Brazil are seeking to diversify farm income and increase production efficiency by integrating livestock grazing of winter annual forages within rotations of summer grain crops. Our objective was to determine grazing and nitrogen fertilization effects on decomposition of winter annual forage residues in corn. A winter cover of oat (Avena strigosa Shreb) and annual ryegrass (Lolium multiflorum L.) was no-till seeded in April 2002 near Ponta Grossa, PR in southern Brazil. Treatment combinations of $\mathrm{N}$ fertilizer ( 0 or $150 \mathrm{~kg} \mathrm{ha}^{-1}$ ) and grazing (grazed or not grazed) were imposed on the winter forage cover. The grazing period was from June to early October 2002. The winter forage was desiccated on 11 October and corn was no-till planted $20 \mathrm{~d}$ later with two $\mathrm{N}$ treatments $\left(0\right.$ and $\left.150 \mathrm{~kg} \mathrm{~N} \mathrm{ha}^{-1}\right)$. Above ground forage biomass residue was hand-clipped, placed in small nylon bags that were scattered on the soil immediately after corn planting, and recovered after five periods of three weeks each for determination of residual dry matter. Results were submitted to analysis of variance. The loss of residual dry matter followed a quadratic function on time. Decomposition of winter annual forage residue was affected by winter treatment $(P<0.02)$, corn $\mathrm{N}$ rate $(P<0.01)$, and by their interaction . At the last collection period ( 132 days after corn planting) differences $(P<0.01)$ were detected only for the winter treatments. Treatments receiving winter-applied $\mathrm{N}$ had greater decomposition of residue regardless of the grazing treatment imposed on the winter forage cover.

Key-words: grazing, nitrogen, decomposition, Zea mays L.
\end{abstract}

\footnotetext{
${ }^{1}$ Enga Agrônoma, Dr. - Departamento de Fitotecnia e Fitossanitarismo- Universidade Federal do Paraná;

${ }^{3}$ Professor PhD Ohio State University - EUA;

${ }^{4}$ Pesquisador Dr. - EMBRAPA

${ }^{5}$ Professor Dr. - Departamento de Plantas Forrageiras e Agrometeorologia - UFRGS.
}

${ }^{2}$ Professor Dr. do Departamento de Fitotecnia e Fitossanitarismo-UFPR - Rua dos Funcionários, 1540, CEP: $80035-50$ - Curitiba - PR.; 


\section{INTRODUÇÃO}

As pastagens apresentam um alto conteúdo de matéria orgânica e densa massa radicular quando bem manejadas, favorecendo a existência de uma grande biomassa microbiana na rizosfera da pastagem (HAYNES e WILLIANS, 1993) que aumenta o seu potencial qualitativo no processo de mineralização. A utilização do sistema integrado da pastagem com a lavoura permite a utilização desses resíduos como cobertura para a semeadura direta de culturas, reduzindo os riscos de erosão do solo e aumentando a reciclagem de nutrientes no sistema. Os animais em pastejo agregam ao sistema quantidades de nutrientes essenciais para as plantas, sendo o nitrogênio $(N)$ um deles. A quantidade de nutrientes retornados via fezes e urina dos animais em pastejo varia amplamente em função da qualidade e quantidade da forragem por eles consumida. Assmann (2001), avaliando a produtividade do milho cultivado em seqüência a uma pastagem (aveia + azevém + trevo branco), fertilizadas com doses de $\mathrm{N}$ e utilizada ou não por meio do pastejo contínuo, encontrou efeito positivo do pastejo sobre a transferência de $\mathrm{N}$ da pastagem para a cultura agrícola. A velocidade de acúmulo de biomassa de uma pastagem pode estar diretamente relacionada com as condições da disponibilidade de nutrientes e com as adubações nitrogenadas, que aumentam o rendimento de matéria seca das plantas forrageiras (WILKINS et al., 2000). Em avaliações de rotação de cultura em área cultivada por meio de plantio direto, há 8 anos, foi possível estimar que cerca de $40 \%$ do $\mathrm{N}$ adicionado ao solo foi incorporado na matéria orgânica (PAVINATO, 1993), razão pela qual seria esperado um aumento na disponibilidade de nitrogênio com o tempo no sistema plantio direto. Os níveis de nitrogênio no solo são determinados basicamente pelo balanço entre quantidade mineralizada a partir da matéria orgânica e da decomposição de resíduos vegetais, da adição de fertilizantes e pelas perdas por lixiviação, volatilização e desnitrificação. Se o pastejo e a adubação nitrogenada afetam a massa seca aérea residual da pastagem, afetará também a sua decomposição porque resultará em uma mudança na qualidade da massa seca. $O$ objetivo deste trabalho foi avaliar a dinâmica da decomposição da fitomassa aérea residual da pastagem de inverno fertilizada ou não com nitrogênio e utilizada ou não por meio de pastejo durante o desenvolvimento da cultura do milho com e sem adubação nitrogenada em um sistema de integração lavoura-pecuária.

\section{METODOLOGIA}

O experimento foi conduzido na Fazenda Escola "Capão da Onça", pertencente à Universidade Estadual de Ponta Grossa - PR, no período de abril de 2002 a maio de 2003. A área encontra-se localizada na região fisiográfica denominada de Segundo Planalto Paranaense, situada entre as coordenadas $25^{\circ} 05^{\prime} 47^{\prime \prime}$ de latitude Sul e $50^{\circ} 03^{\prime} 11^{\prime \prime}$ de longitude Oeste, com altitude média de 1025 m (Fundação Instituto Agronômico do Paraná, 1994). O clima é do tipo cfb, subtropical, e o solo classificado como Argissolo Distrófico. Os tratamentos corresponderam a pastagem de inverno (aveia preta + azevém) fertilizada ou não com nitrogênio e utilizada ou não por meio de pastejo, todos seguidos por cultura de verão (milho) fertilizada ou não com 150 kg.ha-1 de nitrogênio $(\mathrm{N})$. Foi avaliada a dinâmica de mineralização da matéria seca da aveia preta + azevém nas parcelas submetidas ou não ao pastejo e adubadas ou não com $150 \mathrm{~kg} \cdot \mathrm{ha}^{-1}$ de nitrogênio. A área se encontrava originalmente com a cultura do feijão, que foi dessecada com Glifosate na dosagem de $1,5 \mathrm{l} / \mathrm{ha}$ mais Assist $0,5 \%$ no dia 10 de abril de 2002. A semeadura do azevém + aveia preta foi realizada por meio de plantio direto no dia 10 de abril utilizando-se $40 \mathrm{~kg}$ ha $^{-1}$ de sementes. Uma adubação nitrogenada de cobertura foi realizada no dia 28/05/ 2002 utilizando-se a uréia como fonte de $\mathrm{N}$ em dosagem equivalente a $75 \mathrm{~kg} \cdot \mathrm{ha}^{-1}$. Uma segunda aplicação foi realizada em 06/07/2002 também na dosagem de $75 \mathrm{~kg} \cdot \mathrm{ha}^{-1}$ de N. A área total do experimento somou $6880 \mathrm{~m}^{2}$. Por ocasião da entrada dos animais foram isoladas 8 parcelas denominadas área sem pastejo $(\mathrm{S} / \mathrm{P})$, das quais a metade não recebeu nitrogênio $(\mathrm{N})$. O delineamento experimental foi o de blocos ao acaso, com quatro repetições, e os tratamentos alocados às unidades experimentais segundo um arranjo de parcelas sub-divididas, totalizando 16 parcelas principais e 32 sub-parcelas que constaram do milho com zero e $150 \mathrm{~kg}^{\mathrm{h}} \mathrm{ha}^{-1} \mathrm{de}$ nitrogênio. As áreas não pastejadas foram isoladas dos animais por meio de cerca elétrica.

No dia 12/06/2002, quando a pastagem apresentava uma massa de forragem de1650 kg.ha-1, os animais foram colocados na área experimental. $O$ método de pastejo empregado foi o contínuo utilizando-se 2 animais traçadores e um número variável de animais reguladores utilizados para ajustar as intensidades de pastejo. A retirada dos animais foi realizada no dia 11/10/2002. No dia 31/10/2002 foi realizada a semeadura do milho em plantio direto utilizando-se o Híbrido-DK 215. Nesse dia foi realizada uma adubação de base, utilizando-se $300 \mathrm{~kg} \cdot \mathrm{ha}^{-1} \mathrm{da}$ formulação 0-20-20 mais $70 \mathrm{~kg}$. ha-1 de uréia nas unidades experimentais onde a aplicação de nitrogênio estava prevista.

Para a análise da palhada, a massa de forragem no interior de um quadrado de ferro de $50 \mathrm{x}$ $50 \mathrm{~cm}$ foi cortada rente ao solo no dia 11 e 12/10/ 2002, e levada para laboratório, onde foi realizada a separação de colmos, folhas e material morto da aveia-preta + azevém e posteriormente colocada em estufa de circulação forçada de ar a $65^{\circ} \mathrm{C}$ por 72 horas. Após pesagem, o material das amostras teve seu tamanho reduzido a frações de $10 \mathrm{~cm}$ e foi homogeinizado. Uma sub-amostra de aproximadamente 15 gramas de cada material foi 
colhida e colocada em sacos de tela de nylon (método "litter bag") medindo $20 \times 20 \mathrm{~cm}$ e malha $2 \mathrm{~mm}$, totalizando 320 sacos (10 sacos/subparcela). No dia $01 / 11 / 2002$ os sacos que continham o material para decomposição foram colocados sobre a superfície do solo e amostragens para avaliação da matéria seca residual foram realizadas sistematicamente a partir dessa data (Tabela 1). Todo o material vegetal recolhido (palhada) foi triturado em um liquidificador industrial e armazenado em tubos plásticos com tampa de rosca para análises posteriores.

TABELA 1 - Descrição das épocas de coleta da palhada de aveia-preta (Avena strigosa Schreb) e azevém (Lolium multiflorum L.) na fazenda escola "Capão da Onça”, Universidade Estadual de Ponta Grossa, PR, 2002/03.

\begin{tabular}{ccc}
\hline Data & Dias & Intervalo \\
\hline $01 / 11 / 2002$ & 0 & - \\
\hline $19 / 11 / 2002$ & 18 & 18 \\
$10 / 12 / 2002$ & 39 & 21 \\
$09 / 01 / 2003$ & 69 & 30 \\
$08 / 02 / 2003$ & 98 & 29 \\
$11 / 03 / 2003$ & 132 & 34 \\
\hline
\end{tabular}

\section{RESULTADOS E DISCUSSÃO}

A decomposição da matéria seca residual da pastagem de inverno revelou um comportamento quadrático ao longo do período experimental para todos os tratamentos. O tratamento que sofreu pastejo e foi adubado com nitrogênio no período de inverno independente do milho ter sido adubado com nitrogênio ou não, apresentou uma decomposição da matéria seca residual mais acelerada em relação aos demais ao final dos 132 dias de experimento (Figura 3), não diferindo apenas do tratamento sem pastejo com nitrogênio no inverno. Ambos apresentaram no final da avaliação (após 132 dias) um resíduo de MS equivalente a $59,4 \%$ e $55,13 \%$ da massa inicial, respectivamente. A adubação nitrogenada na cultura do milho não resultou em alteração na decomposição da massa seca aos 132 dias, (Figura 4). Esses resultados revelam maior efeito do nitrogênio aplicado no período do acúmulo da massa seca durante o inverno sobre o processo de decomposição da massa residual da pastagem, alterando inclusive a sua constituição. A presença de uma maior relação colmo/ folha (Figura 1 e 2) e maiores quantidades de material morto na massa seca antecedendo a dessecação podem explicar a redução da taxa de mineralização da matéria orgânica provavelmente pela diminuição da atividade microbiana. Pode-se deduzir dessas informações que a reciclagem de nutrientes tem uma dependência direta de todos esses fatores. O pastejo também é capaz de transformar uma grande parte da biomassa aérea produzida em dejetos, que terão uma forma de reciclagem dos nutrientes diferente daquela que ocorre quando esse processo passa pela degradação da palhada.

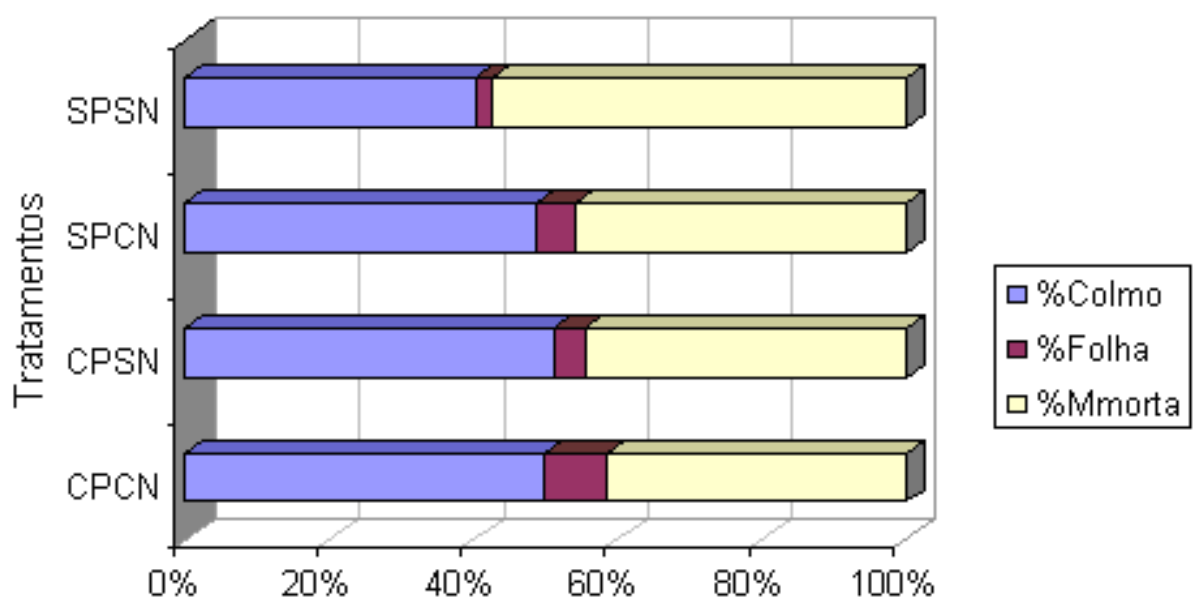

Percentagem

FIGURA 1 - Constituição da massa residual da pastagem (\%) em termos de colmo, folha e material morto nos tratamentos sem pastejo sem N (SPSN); sem pastejo com N (SPCN); com pastejo sem N (CPSN) e com pastejo com N (CPCN). 
LANG, C.R. et al. Fitomassa aérea residual da...

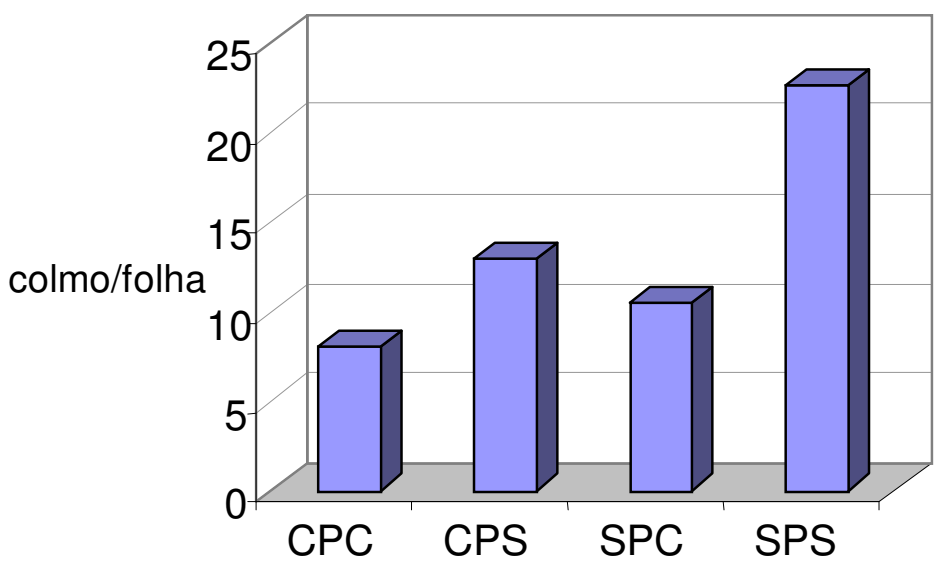

FIGURA 2 - Constituição da massa residual da pastagem quanto à relação colmo/folha nos tratamentos sem pastejo sem N (SPSN); sem pastejo com N (SPCN); com pastejo sem N (CPSN) e com pastejo com N (CPCN).

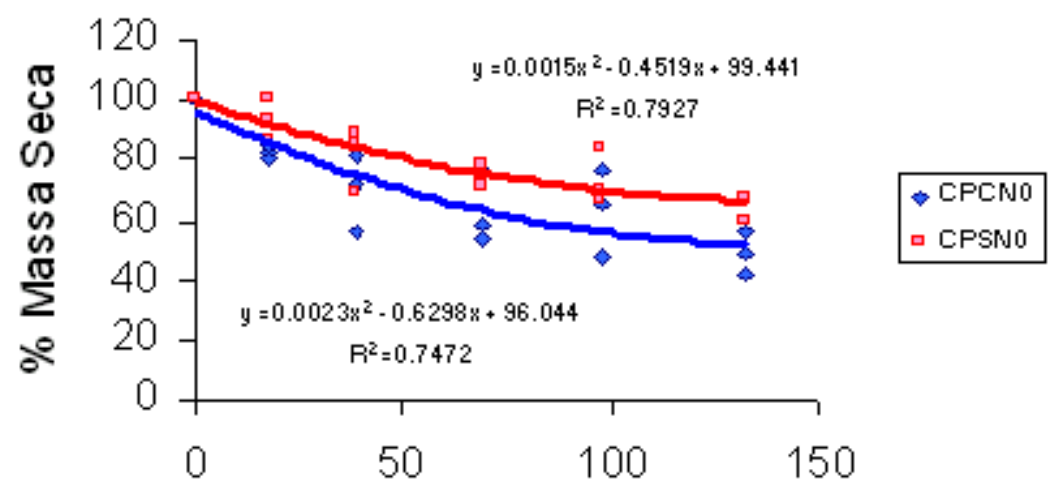

Dias

FIGURA 3 - Decomposição da massa seca residual da pastagem de aveia preta e azevém com pastejo e com e sem adubação nitrogenada seguida de cultura do milho sem nitrogênio, Fazenda "Capão da Onça”, Ponta Grossa, PR, 2003.

Os resultados deste experimento corroboram aqueles de Bauer et al. (1987), que confirmaram o aumento da taxa de reciclagem do $\mathrm{N}$ por meio da deposição de urina e fezes, muito embora nas áreas com pastejo tenha ocorrido um menor acúmulo de matéria seca residual relativamente às áreas não pastejadas.

Os tratamentos que não foram pastejados e receberam adubação nitrogenada no período de inverno, com ou sem nitrogênio na cultura subseqüente do milho, apresentaram uma decomposição semelhante (Figuras 5 e 6), partindo inicialmente de $7.000 \mathrm{~kg} \cdot \mathrm{ha}^{-1}$ de matéria seca residual e, após 132 dias apresentando cerca de $2400 \mathrm{~kg} \cdot \mathrm{ha}^{-1}$, equivalente a uma decomposição de $59 \%$. Por outro lado, os tratamentos sem nitrogênio no inverno apresentaram uma decomposição maior quando receberam adubação nitrogenada de $150 \mathrm{~kg} \cdot \mathrm{ha}^{-1}$ na cultura subseqüente do milho, cerca de $51,1 \%$. 


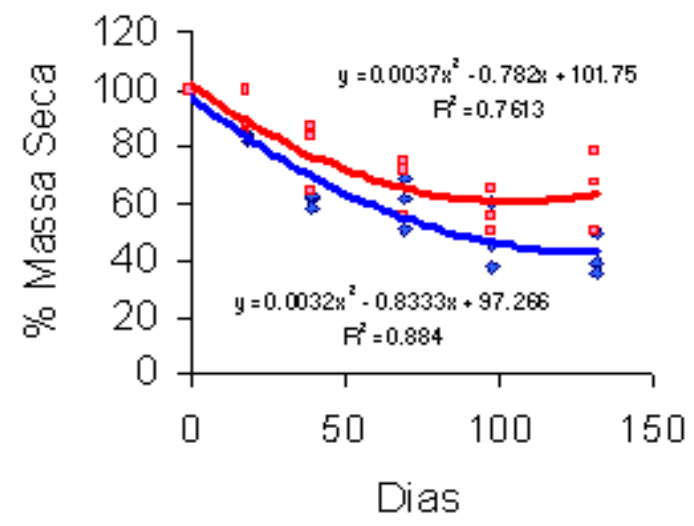

FIGURA 4 - Desaparecimento da massa seca residual da pastagem de aveia preta e azevém com e sem adubação nitrogenada e com pastejo e a cultura do milho com $150 \mathrm{~kg} \cdot$ ha $^{-1}$ de nitrogênio, Fazenda "Capão da Onça”, Ponta Grossa, PR, 2003.

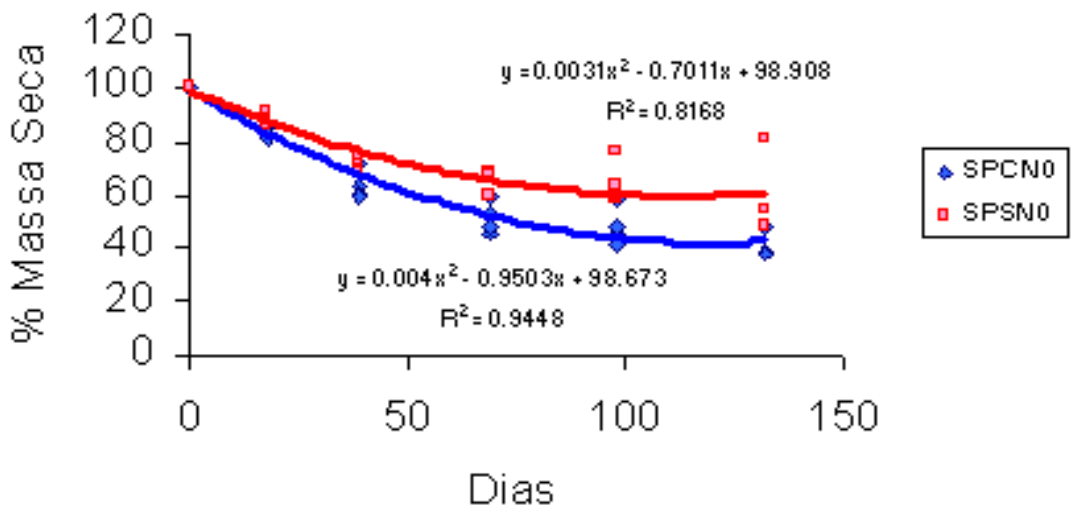

FIGURA 5 - Desaparecimento da matéria seca residual da pastagem de aveia preta e azevém com e sem adubação nitrogenada e sem pastejo no desenvolvimento da cultura do milho sem nitrogênio, Fazenda "Capão da Onça”, Ponta Grossa, PR, 2003.

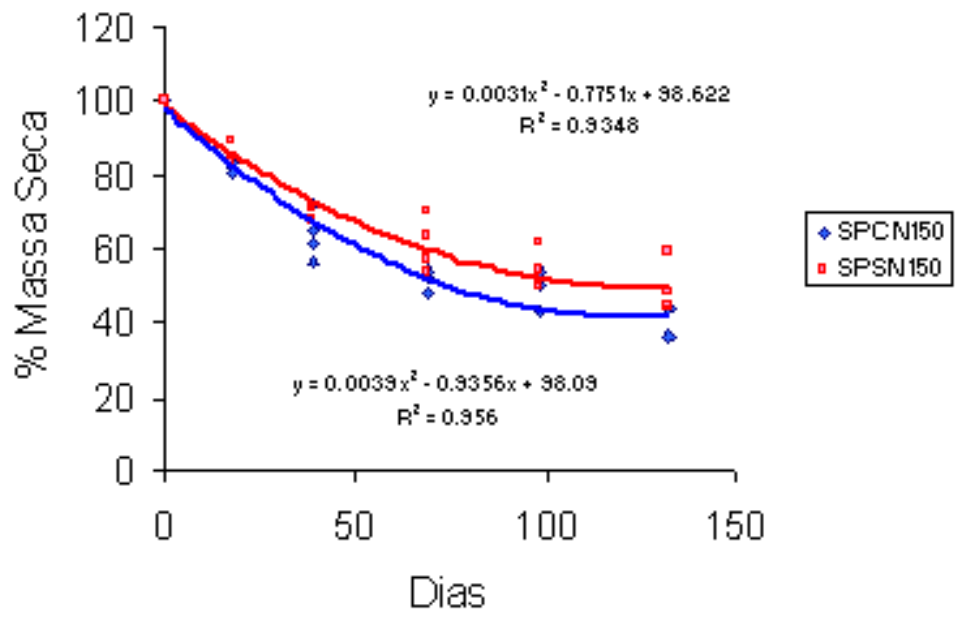

FIGURA 6 - Desaparecimento da matéria seca residual da pastagem de aveia preta e azevém com e sem adubação nitrogenada e sem pastejo no desenvolvimento da cultura do milho com $150 \mathrm{~kg}_{\text {.ha-1 }}$ de nitrogênio, Fazenda "Capão da Onça”, Ponta Grossa, PR, 2003. 
LANG, C.R. et al. Fitomassa aérea residual da...

A importância do uso de adubação nitrogenada no período de inverno está em possibilitar um maior acúmulo de matéria seca residual e, ao mesmo tempo, garantir uma maior decomposição do resíduo. No presente experimento, (Tabela 2), isso correspondem a uma massa residual de 7093 kg.ha-1 que, após 132 dias de avaliação, resultou em apenas $2887 \mathrm{~kg} \cdot \mathrm{ha}^{-1}$, equivalente a uma decomposição de $59,4 \%$. Nas situações onde não houve aplicação de N no inverno, a decomposição foi de $40,7 \%$, sendo que o total de matéria seca inicial (5678 kg.ha-1), mesmo sendo inferior, resultou em um maior resíduo (3341 kg.ha-1).

TABELA 2 - Proporção (\%) da matéria seca residual da mistura aveia-preta + azevém coletadas no "litter bag" aos 132 dias de avaliação, Fazenda "Capão da Onça", Ponta Grossa, PR, 2003.

\begin{tabular}{cc}
\hline TRATAMENTOS & MÉDIAS ${ }^{*}$ \\
\hline CPSN & 54,6 a \\
SPSN & 54,2 a \\
CPCN & 44,9 ab \\
SPCN & 40,6 b \\
\hline
\end{tabular}

Coeficiente de Variação $(\%)=11.9$. Médias seguidas da mesma letra não diferem significativamente para os tratamentos pelos Teste de Duncan $(p>0,05)$.

\section{CONCLUSÃO}

A decomposição dos resíduos da mistura de aveia preta + azevém foi aumentada pela aplicação de nitrogênio durante o período de inverno tanto nas áreas com ou sem pastejo. A adubação nitrogenada na pastagem de inverno na dose de $150 \mathrm{~kg} \cdot \mathrm{ha}^{-1}$ permitiu aumento da fitomassa e de sua qualidade (menor relação $\mathrm{C} / \mathrm{N}$, maior \% de folhas e menor relação colmo/folha).

\section{REFERÊNCIAS}

ASSMANN, T.S. Rendimento de milho em áreas de integração lavoura-pecuária sob o sistema de plantio direto, em presença e ausência de trevo branco, pastejo e nitrogênio. Curitiba, 2001. 80p. Tese ( Doutorado em Agronomia - Produção Vegetal) Setor de Ciêcias Agrárias, Universidade Federal do Paraná, 2001.

BAUER, A.; COLE, C. V. e BLACK, A . L. Soil property comparisons irvirgin grassland between grazed and nongrazed management systems. Soil Science Soc. Am. J., Madison, 51:176-182, 1987.

BOCOCK, K. L. and GILBERT, O . The disappearance of leaf litter under different woodland conditions. Plant and Soil. V.9, 1957. p. 179-185.

HAYNES, R. J.; WILLIAMS, P. H. Nutrient cycling and soil fertility in the grazed pasture ecosystem. Advances in Agronomy, New York, v.49, p.119-199, 1993.

IAPAR - Instituto Agronômico do Paraná. Cartas Climáticas do Estado do Paraná, 1984. Londrina:IAPAR, 1994. 49p.

PAVINATO, A. Teores de carbono e nitrogênio do solo e produtividade de milho afetado por sistemas de culturas. Porto Alegre, 1993, 122 f. Dissertação (Mestrado em área de concentração Ciência do Solo) - Universidade Federal do Rio Grande do Sul. WILKINS, P. W.; ALLEN, D.K.; MYTTON, L.R. Differences in the nitrogen use efficiency of perennial ryegrass varieties under simulated ratational grazing and their effects on nitrogen recovery and herbage nitrogen content. Grass and forage science, v. 55, n. 1, p. $69-76$, 2000.

Recebido em 10/03/2004 Aceito em 06/06/2005 